\title{
1.3 交互性强
}

智能电网建设与传统的电网相比较而言, 更加的重视用户的使用体验, 电力企业中的管理部门在通过电网与用户 进行沟通的过程当中, 能够根据用户给予的反馈, 对电网进行具有针对性的调整, 通过不断对电网进行优化, 提升自 身的工作效率, 为人们提供更好的服务。(四) 自动化水平高在我国现阶段的智能电网建设过程中, 已经让电网具有了 自动排除障碍以及自动修复的功能, 这让电网在实际运行中的安全性以及稳定性都有了非常明显的提高, 更好的保证 了我国电网的运行质量, 同时也降低了工作人员的工作量, 缩短了实际运行以及维护工作的时间, 在很大程度上降低 了企业成本的投入。

\section{2 在智能电网建设过程中运用电力工程技术的原因}

\section{1 有利于提高智能电网整体的效率}

通过有效提升智能电网的效率, 充分将电力工程技术应用到智能电网建设中。使智能电网实现了自动化控制。同时 还可以明确采集的数据。除此之外, 可以全面提升电网的运行效率 ${ }^{[1]}$ 。

\section{2 提升电网数据采集能力}

传统的电网技术水平并不是很高。并不能具备自动化和智能化。同时运行效率普遍偏低。对于收集的数据信息不 能完成自动化划分。当前, 智能电网运用先进的技术, 可以将电网设备数据自动化收集, 同时进行自动划分。在电网建设 的技术投入和系统完善方面都有所改善。确保电网运行的高效性和便利性。

\section{3 电力工程具体技术}

\section{1 质量优化技术}

质量优化技术主要是通过对电能进行等级的划分而建立起科学的、完善的电能质量评估体系, 方便工作人员对用 电的经济性来进行分析。通过数据的分析构建起一个合理的质量评估系统。在我国现阶段, 电力市场的竞争是非常激 烈的, 电力企业想要取得更好的发展, 就必须通过自身不断的完善来提高电网的质量, 提高其经济性以及效率。相关 工作人员可将自适应静止无功补偿技术、连续调谐滤波器技术、源滤波器技术应用在电网建设当中, 能够更好的提高 电网的质量。上述技术不但成本比较低, 更是具有环保性以及低能耗的性质, 具有较好的应用前景 ${ }^{[2]}$ 。

\section{2 柔性交流输电技术}

将柔性交流输电技术应用在智能电网的建设当中, 能够起到扩大清洁能源应用范围的作用, 更好的落实电力技术 与通信控制技术与微电子技术一体化, 让工作人员能够更加灵活的对交流电进行控制。现阶段我国在智能电网建设的 过程当中, 建设的主要目的是输送高压输变电, 为了能够降低电力在运行过程中对于环境的影响, 相关工作人员通过 应用清洁能源的方式来更好的进行能源的隔离。将柔性交流输电技术与智能电网更好的结合在一起, 因其更加符合可 持续发展理念将会是未来的一种发展趋势 ${ }^{[3]}$ 。

\section{3 高压直流输电技术}

我国现阶段广泛采用的是直流输电方式。因此相关工作人员应该重视高压直流输电技术在我国电网建设当中的应 用。同时, 将环路器应用在直流输电系统当中, 能够起到让电流更加稳定、输电工作更具安全性、可靠性的作用, 从 经济层面来说, 其性价比也是比较高的。值得相关工作人员需要注意的是, 将高压直流输电技术应用在电网建设当中 能够起到远距离输电的作用, 能够让山区中的人们得到稳定的电力供应, 提高山区人们的生活质量, 并起到提高全国 范围内用电安全性及稳定性的作用。

\section{4 电力工程技术在智能电网建设中的应用}

\section{1 在电源中的应用}

在整个电力工程中, 电源装置能够为整个系统的运行提供充足的能源, 将电力工程技术加以切实运用, 可以有效 的为系统提供各类不同的店员, 从而为系统的实际需要提供帮助。诸如: 在针对智能电网的运行情况实施监控的时候, 通常都会利用专业的高频开关店员来辅助电子设备工作。在蓄电池进行充电的时候, 利用直流电源, 能够为变电所的 正常运行提供稳定的能源。

\section{2 在发电中的应用}

电力工程技术可以借助基础设备来将其他种类的能源转变为电能, 从而弥补电网发电设备不充足的问题, 并且可 以对耗电量实施专门的检测和控制, 提升机电设备的利用效率。当前, 无功发电技术以及电气传动技术都取得了显著 
的进步, 从而被人们大范围的引用到了智能电网之中。

\section{3 在输电中的应用}

要想保证电网运行的稳定性和高效性, 需要针对智能电网发电以及运输电能环节加以切实的管控, 这项工作对于 设备和数学的分析能力要求相对较高。在实施电力运输的时候, 整个电网中的线路往往会遭到外界各种因素的影响, 极易引发故障问题。智能电网的大范围运用有效的解决了以往陈旧的物理电网中能量损耗较大的问题, 可以自行结合 电网输电情况来对功率进行调整, 提升电能传输的稳定性 ${ }^{[4]}$ 。

\section{4 在配电环节的应用}

现如今, 在我国使用最为频繁的是功能较少的传统数字变电站, 其作用就是针对电力输送变换信息进行切实的监 督管控。在整个智能电网中, 电力工程技术能够实现对所有信息数据的监测和收集, 并且可以完成针对电力系统的管 控和保护, 促进电网运行的效率, 从而完成配电网络的双向流动和输送终端的电压自动调控 ${ }^{[5]}$ 。

\section{5 结语}

智能电网是我国科学技术水平不断提升的产物, 因为具有良好的优越性, 所以广泛的受到了人们的青睐。在针对 智能电网实施建设工作的时候, 务必要严格遵从相关法律法规, 大范围的引入最前沿的技术, 促进智能电网建设质量 和效率的不断提升, 从根本上确保电网运行的效率。其次, 好需要加大力度来对智能电网建设工作进行优化和创新, 从而提升智能电网建设的整体水平, 确保我国电力行业的健康发展。

\section{[参考文献]}

[1]王祝军, 宋安心. 电力工程技术应用于智能电网建设中的分析 [J]. 科技风, 2019(31): 182 .

[2]吴雷, 于志强. 电力工程技术应用于智能电网建设中的分析 $[J]$. 科技风, 2019 (15) : 170 .

[3]沈忱. 电力工程技术在智能电网建设中的应用 $[\mathrm{J}]$. 通信电源技术, 2018, 35(07) : 136-137.

[4]郑豪涁. 智能电网建设中电力工程技术的应用 $[\mathrm{J}]$. 科技资讯, 2017, 15 (32) : 47-48.

[5]雷中平. 电力工程技术在智能电网建设中的应用初探 $[\mathrm{J}]$. 科技风, $2017(16): 229$.

作者简介: 王宇 (1987-), 女, 合肥工业大学, 材料学专业, 工程管理, 工程师。孔维权 (1988-), 男, 中国科学技 术大学, 材料物理与化学, 变电设计, 工程师。 